\title{
G.N. Fursey
}

Intensive electron emission in a strong electric field in vacuum nanoelectronics and highpower electronics

ABSTRACT. This paper briefly discusses the remarkable characteristics of the processes of field electron (FE) and explosive electron (EE) emission in a strong electric field. Special emphasis is placed on the recently discovered extraordinary fundamental effects occurring at carbon nanoclusters: the low-threshold FE and EE. Important possible practical applications of these phenomena are considered, including the opportunity to develop a new type of very compact Xray devices.

Keywords: explosive emission, field emission, graphene, high-current electronics, low threshold field emission, nanotubes, portable $\mathrm{X}$-ray devices, vacuum nanoelectronics

Nanotechnology Perceptions 9 (2013) 167-185

Nonsubscribers: purchase individual article 